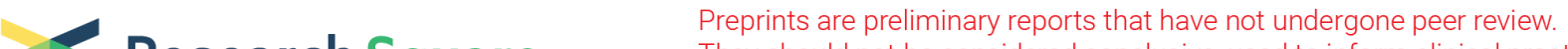 $\begin{array}{ll}\text { Research Square } & \text { They should not be considered conclusive, used to inform clinical practice, } \\ \text { or referenced by the media as validated information. }\end{array}$ \\ Seeds of phase transition to thermoacoustic instability
}

Raghunathan Manikandan

Nitin Babu George ( $\nabla$ nitinbabunit@gmail.com )

Potsdam Institute for Climate Impact Research https://orcid.org/0000-0003-4123-6944

Vishnu Unni

University of California San Diego https://orcid.org/0000-0002-8830-9779

R. Sujith

Indian Institute of Technology Madras https://orcid.org/0000-0002-0791-7896

Jürgen Kurths

Potsdam Institute for Climate Impact Research https://orcid.org/0000-0002-5926-4276

Elena Surovyatkina

Potsdam Institute for Climate Impact Research https://orcid.org/0000-0001-5136-4988

\section{Article}

Keywords: thermoacoustic instability, emissions, phase transition

Posted Date: August 26th, 2021

DOl: https://doi.org/10.21203/rs.3.rs-744803/v1

License: (c) (1) This work is licensed under a Creative Commons Attribution 4.0 International License.

Read Full License 


\title{
Seeds of phase transition to thermoacoustic instability
}

\author{
Manikandan Raghunathan ${ }^{1}$, Nitin B. George ${ }^{2,4, *}$, Vishnu R. Unni ${ }^{3}$, R. I. Sujith ${ }^{1}$, \\ Juergen Kurths ${ }^{2,4}$, and Elena Surovyatkina ${ }^{2,5}$ \\ ${ }^{1}$ Department of Aerospace Engineering, Indian Institute of Technology, Madras 600036, India \\ ${ }^{2}$ Potsdam Institute for Climate Impact Research, P.O. Box 601203, 14412 Potsdam, Germany \\ ${ }^{3}$ Department of Mechanical and Aerospace Engineering, University of California, San Diego, USA \\ ${ }^{4}$ Department of Physics, Humboldt University of Berlin, Berlin, Germany \\ ${ }^{5}$ Space Research Institute of Russian Academy of Sciences, Moscow, Russia \\ *Corresponding author, george@pik-potsdam.de
}

Tackling the problem of emissions is at the forefront of scientific research today. While industrial engines designed to operate in stable regimes produce emissions, attempts to operate them at "greener" conditions often fail due to thermoacoustic instability. During thermoacoustic instability, hazardous high amplitude periodic oscillations lead to failure of these engines in power plants, aircrafts and rockets. Yet, identifying the onset of thermoacoustic instability remains elusive due to spatial variability and the continuous evolution of spatiotemporal patterns in the reacting flow field. Here, we show experimental evidence of early manifestation of the onset of thermoacoustic instability at certain zones. Our findings allow us to identify a critical threshold that enables us to distinguish stable operating regimes from hazardous operations. This opens new perspectives for predicting the onset of thermoacoustic instability and could be a step forward to "greener" operations. The developed methodology is applicable for other systems exhibiting phase transitions.

All combustion engines in automobiles, airplanes, ships etc. produce emissions. But industrial systems produce more emissions such as oxides of nitrogen and sulphur due to high flame temperatures. To reduce these emissions and operate in "greener" conditions, we need to lower the flame temperatures. Increasing the proportion of air to the fuel reduces the flame temperature. However, such "greener" operations often result in the dangerous phenomenon of thermoacoustic instability, which results in large amplitude oscillations leading to catastrophic consequences. Controlling the occurrence of thermoacoustic instability creates opportunities to operate under "greener" conditions. 
But the major problem is in identifying the onset of thermoacoustic instability. This is a problem that has been documented in various systems, but still remains a challenging one ${ }^{1-8}$. Thermoacoustic instability occurs because of the coupling between the flame and the acoustics field within the combustion chamber ${ }^{5}$ reinforced by emerging large coherent structures or vortices in the flow field ${ }^{9,10}$.

Turbulent flow systems are characterized by chaotic or disordered behaviour. However, at certain conditions they have organized flow structures ${ }^{11-15}$. The emergence of such ordered spatiotemporal patterns is ubiquitous in ecology ${ }^{16,17}$, climate $^{18,19}$, chemistry ${ }^{20-22}$, biology ${ }^{16,23,24}$, engineering ${ }^{23,25}$, etc. Such regularities in the spatiotemporal behaviour appear near a phase transition, when certain system parameters move beyond a critical point ${ }^{26-28}$. This phase transition demarcates two contrasting regimes of the system. However, identifying the impending phase transition is quite often a challenge, since there is no visible change in the dynamics as the system approaches the critical point.

Recently, progress has been made towards the solution of identifying the phase transition, wherein some studies on spatiotemporal systems show that while a phase transition occurs for the whole system, there may be regions where such transitions emerge earlier. In a study on the Indian monsoon prediction, Stolbova et al. ${ }^{29}$ uncovered two unique locations in the Indian subcontinent (referred to as tipping elements), where critical conditions originate and then propagate in a certain direction that results in the transition from pre-monsoon to monsoon; the regularities between the tipping elements allows a long term prediction of the monsoon onset. Rocha et al. ${ }^{30}$ showed that an interaction between tipping elements can result in cascading effects and a phase transition in the global dynamics. In short, it is necessary to locate such zones to identify the origin of the phase transition. In this paper, we focus on the identification of such zones in a turbulent combustion system. We utilize the phenomenon of critical growth of fluctuations ${ }^{31,32}$ as an indicator of the onset of such a phase transition.

In particular, we investigate the spatiotemporal dynamics in a confined reactive turbulent system when it approaches the state of thermoacoustic instability $\left(S_{T I}\right)$. In this system, flow perturbations cause heat release rate fluctuations from the flame, which subsequently generates sound waves. Next, these sound waves get reflected from the boundaries back to the source and further modify the heat release rate fluctuations. This process results in a positive feedback, when the acoustic pressure fluctuations due to the sound waves and the unsteady heat release rate become in phase ${ }^{33}$. Such a positive feedback favours the growth of acoustic oscillations, leading to periodic temporal dynamics ${ }^{5-8}$ and the periodic emergence of spatially organized patterns ${ }^{34-37}$. Notably, the dangerous high amplitude periodic oscillations of acoustic pressure during $S_{T I}$ cause significant losses to the power and propulsion industry by causing structural failure, damaging the navigation and control system, overwhelming the thermal protection system, etc. ${ }^{6,8,35}$. The onset of periodic oscillations $\left(S_{T I}\right)$ occurs via a transition from a state of chaotic fluctuations $\left(S_{C}\right)$, through the state of intermittency $\left(S_{I N}\right)^{38-40}$. 
Different spatiotemporal patterns characterize each state of the system. It was recently discovered that $S_{C}$, referred to as combustion noise, is chaotic ${ }^{41,42} . S_{C}$ is also characterized by disorganized flow and flame dynamics ${ }^{41,43-45}$. During $S_{I N}$, bursts of periodic oscillations appear amidst epochs of aperiodic dynamics at apparently random intervals ${ }^{39}$. Additionally, during $S_{I N}$, macroscopic patterns at the size of the system geometry emerge during the regime of periodic oscillations, while disordered small-scale structures exist during the regime of aperiodic oscillations ${ }^{43-45}$. On the other hand, $S_{T I}$ typically exhibits the coexistence of large-scale coherent flow structures or vortices, spatially and temporally organized reaction fields, distinct standing wave modes, large-amplitude periodic acoustic pressure oscillations etc ${ }^{44}$. In fact, during the transition from $S_{C}$ to $S_{T I}$ via $S_{I N}$, the disordered dynamics decreases continuously, while the ordered dynamics increases through the collective behaviour of small-scale structures ${ }^{44,45}$. This continuous evolution makes it difficult to identify the critical point of the system parameter or the onset of $S_{T I}$, which is crucial for designing safe operating regimes of such complex spatiotemporal systems. Further, whether the onset of $S_{T I}$ can be forewarned has been a vital research question that has been pursued in the recent decades ${ }^{46-54}$

The present study is the first attempt to identify the onset of thermoacoustic instability that is not based on user-defined thresholds. To that end, we take a step towards identifying zones where conditions for the phase transition originate. Following the approach of Stolbova et al. ${ }^{29}$, we utilize the variance of fluctuations of system observables (flame fluctuations) at different locations across the spatial domain of the thermoacoustic system to find zones with maximum growth in the variance of fluctuations, unraveling the seeds of the phase transition. Furthermore, we identify emerging longrange correlations in the flame fluctuations between these zones well before $S_{T I}$. Finally, we analyse the flame fluctuations at these zones to define the onset of $S_{T I}$ and predict it in advance. We conjecture that such an approach will be helpful to identify the earliest manifestation of phase transitions and predict them in other engineering and natural systems as well.

\section{Analysis of fluctuations in the global observables}

To investigate the transition from $S_{C}$ to $S_{T I}$ via $S_{I N}$, we perform experiments on a laboratory scale turbulent combustor ${ }^{39,55-58}$ and acquire spatiotemporal data. Our study analyses the transition between the dynamical states by continuously varying a system parameter, the air mass flow rate $\left(\dot{m}_{\text {air }}\right.$ ) from low to high values ${ }^{47,59-62}$. In all the experiments, we increase $\dot{m}_{\text {air }}$ from $537 \pm 8$ SLPM (SLPM denotes standard liters per minute) to $780 \pm 10$ SLPM linearly with time. This change in $\dot{m}_{\text {air }}$ corresponds to an increase in the Reynolds number $R e$ from $1.45 \times 10^{4} \pm 428$ to $2.05 \times 10^{4} \pm 555$. We measure two variables that represent the global state of the system: i) the acoustic pressure fluctuations $p^{\prime}(t)$ and ii) the global heat release rate $\dot{Q}(t)$ at a data acquisition rate of $10 \mathrm{kHz}$. Meanwhile, we 
acquire spatiotemporal data of the local heat release rate $\dot{q}(\mathbf{x}, t)$ through high-speed imaging of $C H^{*}$ radical in the combustion zone at a data acquisition rate of 500 frames per sec and a spatial resolution of $800 \times 600$ pixels. (More information on the measurements, data acquisition systems, experimental uncertainties and experimental setup can be found in Methods). Furthermore, we perform seven independent experiments keeping the same conditions of system parameters.
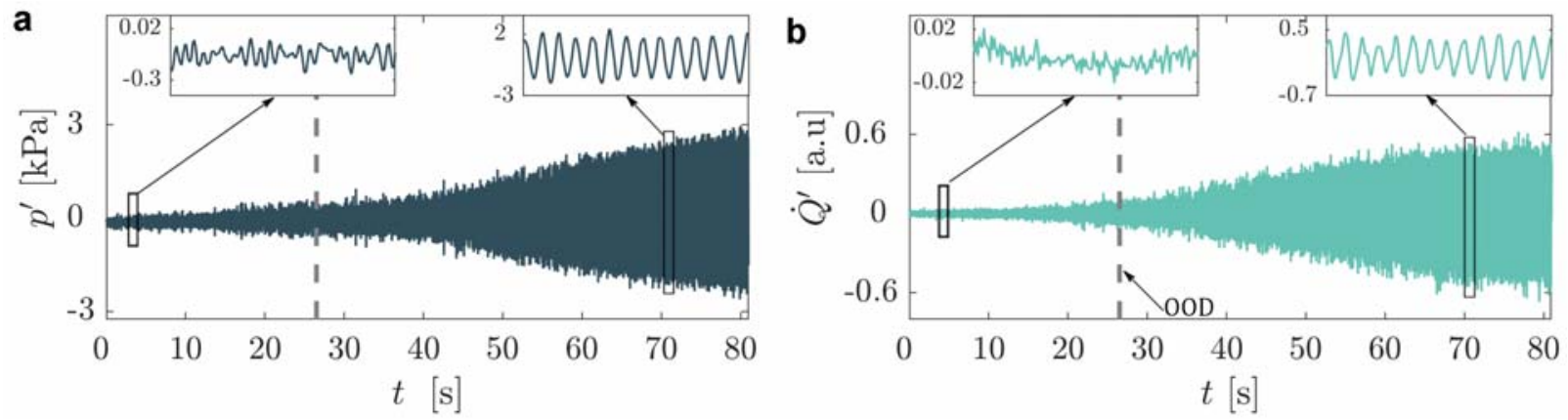

Figure 1: Transition from low amplitude chaotic fluctuations to high amplitude periodic oscillations in a turbulent thermoacoustic system. The time series of (a) $p^{\prime}(t)$ and (b) $\dot{Q}^{\prime}(t)$. The time series are shown for continuous variation of $\dot{m}_{\text {air }}$ from 537 SLPM at $t=0$ to 780 SLPM at $t=81$ s. The insets in a and $\mathbf{b}$ show zoomed-in views of $p^{\prime}(t)$ and $\dot{Q}^{\prime}(t)$ during $S_{C}$ and $S_{T I}$. We mark the point of the onset of ordered dynamics (OOD) (Refer to methods). At the OOD, $\dot{m}_{\text {air }}$ and $R e$ are 617 SLPM and 16380 respectively.

At the outset, we analyze the acoustic pressure and global heat release rate fluctuations, $p^{\prime}(t)$ and $\dot{Q}^{\prime}(t)$ respectively. As we increase $\dot{m}_{a i r}, p^{\prime}(t)$ (Fig. 1a) and $\dot{Q}^{\prime}(t)$ (Fig. 1b) change from aperiodic fluctuations to periodic oscillations. It is important to note that $p^{\prime}(t)$ and $\dot{Q}^{\prime}(t)$ are variables that indicate the global state of the system and are only functions of time. At the beginning of the experiment $(t=0) p^{\prime}(t)$ and $\dot{Q}^{\prime}(t)$ are aperiodic and have low amplitude (see picture insets near $t=5$ s). Such fluctuations show the chaotic temporal behaviour of the system at low values of $\dot{m}_{\text {air }}$ during the occurrence of $S_{C}{ }^{41}$. In contrast, as $\dot{m}_{a i r}$ increases, the fluctuations become periodic and grow in amplitude (picture insets at $t=70 \mathrm{~s}$ ). The dominant frequency in $p^{\prime}(t)$ for the large amplitude periodic oscillations, estimated by the Fast Fourier Transform (FFT) of a 1 second time window between $t=$ $79 \mathrm{~s}$ and $80 \mathrm{~s}$ is $135 \mathrm{~Hz}$ (uncertainty of $0.61 \mathrm{~Hz}$ ). This frequency nearly corresponds to the fundamental frequency of a quarter wave acoustic mode in the combustion duct. However, neither $p^{\prime}(t)$ nor $\dot{Q}^{\prime}(t)$ show a demarcation between the aperiodic state and periodic state of the thermoacoustic system because of the continuous growth of periodicity.

\section{Critical phenomena prior to the phase transition from $S_{C}$ to $S_{T I}$}

We utilize an indicator of phase transitions, the variance of fluctuations $\left(\sigma^{2}\right)$ to reveal critical phe- 


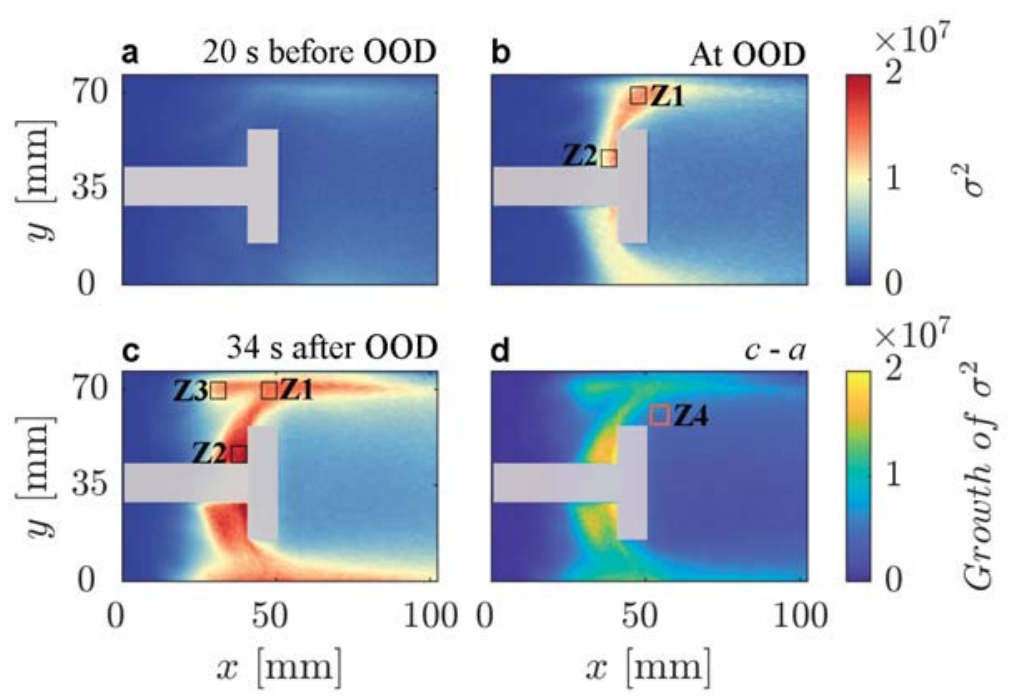

Figure 2: Critical phenomena in the local heat release rate fluctuations precede the state of thermoacoustic instability. The variance of fluctuations $\left(\sigma^{2}\right)$ of the local heat release rate at different instants (a) $20 \mathrm{~s}$ before the OOD, (b) at the OOD and (c) $34 \mathrm{~s}$ after the OOD. At the center of the images, the bluff body and its shaft are marked with a grey color. The bluff body creates a low-velocity region to stabilize the flame in the combustor. Behind the bluff body is the wake, a recirculation zone formed by the fluid moving past the bluff body. $\sigma^{2}$ increases at certain regions with a high magnitude, marked by black squares and referred to as zone $1(\mathrm{Z} 1)$, zone $2(\mathrm{Z} 2)$ and zone $3(\mathrm{Z} 3)$. Z1 is above the bluff body, Z2 is at the corner between the bluff body and the shaft while Z3 is upstream of the bluff body and near the combustor wall. At Z1 and Z2, $\sigma^{2}$ increases before the OOD, while at Z3, $\sigma^{2}$ increases after OOD. $\mathbf{d}$ indicates the growth of $\sigma^{2}$, calculated as the difference of $\sigma^{2}$ between two-time instants at $t=5.5 \mathrm{~s}$ and $t=59.5 \mathrm{~s}(\mathbf{d}: \mathrm{c}-\mathrm{a})$. The phase transition ceases to exist in the wake, as observed by the lack of growth in $\sigma^{2}$ in $\mathbf{d}$. We also mark a reference zone (Z4) by an orange square in $\mathbf{d}$ at a region between the wake of the bluff body and Z4. Thus, Z4 indicates a boundary between regions where the phase transition occurs (at Z1, Z2 and Z3) and where it does not (the wake).

nomena in the local heat release rate fluctuations $\left(\dot{q}^{\prime}(\mathbf{x}, t)\right)$. In this study, $\dot{q}^{\prime}(\mathbf{x}, t)$ is the only variable where we have spatial information. In fact, the spatial integral of $\dot{q}^{\prime}(\mathbf{x}, t)$ gives the global measure $\dot{Q}^{\prime}(t)$. We calculate $\sigma^{2}$ of $\dot{q}^{\prime}(\mathbf{x}, t)$ at each pixel in the images. Following this, we estimate the composite averages of the $\sigma^{2}$ field by identifying the onset of ordered dynamics (OOD) for each experiment. We utilize the composite averages because the OOD need not occur at the same point in time across different experiments. More details on the calculation of $\sigma^{2}$ of $\dot{q}^{\prime}(\mathbf{x}, t)$, composite averages and the identification of OOD are described in Methods. Figures 2a-c show the composite averages of $\sigma^{2}$ at various time instants with OOD as the time reference. The composite averages show a localized increase in $\sigma^{2}$ as the system approaches OOD. 
The non-uniform distribution of $\sigma^{2}$ before OOD occurs due to non-uniform flow conditions associated with the geometry of the bluff body and the resultant non-uniform heat release rate. $\sigma^{2}$ at all regions is low much before the OOD (Fig. 2a). However, with an increase in $\dot{m}_{\text {air }}$ and near OOD, $\sigma^{2}$ increases particularly at two zones 1 and 2, referred to as Z1 and Z2 (Fig. 2b). Additionally, at these locations, $\sigma^{2}$ remains high relative to other regions long after the OOD (Fig. 2c). On the other hand, the recirculation zone behind the bluff body has low $\sigma^{2}$ (Figs. 2a-c) because of aperiodic fluctuations (shown later in Fig. 3d). After the OOD, we observe a third zone, Z3, which shows an increase in $\sigma^{2}$ (Fig. 2c). In general, figure $2 \mathrm{~d}$ shows that $\mathrm{Z} 1, \mathrm{Z} 2$ and $\mathrm{Z} 3$ exhibit the highest growth of $\sigma^{2}$. We find that the increase in $\sigma^{2}$ at the Z1, Z2 and Z3 is statistically significant at $99.9 \%$ (see supplementary Fig. 8). We mark a region Z4 by an orange square between $\mathrm{Z} 1$ and the wake region (Fig. 2d), which shows low growth in $\sigma^{2}$. Due to the combustor's axisymmetric profile with respect to the shaft, we have similar dynamics below the shaft. Thus, the growth in $\sigma^{2}$ of the flame fluctuations allows us to locate Z1, Z2 and Z3, out of which Z1 and Z2 exhibit the earliest manifestation of the onset of thermoacoustic instability.

The increase of $\sigma^{2}$ within the confined reactive system indicates that critical phenomena occurs at selected zones of the flame enroute to $S_{T I}$. The geometry of the system gives special importance to different regions within the confined reactive system. For instance, flame stabilization occurs at the bluff body. The corner between the shaft and the bluff body where $\mathrm{Z} 2$ is located provides a lowvelocity region, vital for flame stabilization ${ }^{4}$. This region is directly in line with the incoming bulk flow of fresh reactants. $\mathrm{Z} 1$ is a location of high heat release rate fluctuations after the impingement of large-scale flow structures while $\mathrm{Z} 3$ is at the region where the largest size of the flow structure exists before impingement ${ }^{44}$. Even though the flame is spatially extended in the confined reactive system, there may be certain zones where the increase in local fluctuations appear regularly due to the geometry of the system. Thus, the fact that criticality does not appear in all regions within the combustor is not surprising.

\section{Local increase in variance of fluctuations as an indicator of the phase transition.}

The increase in the variance of fluctuations of $\dot{q}^{\prime}(t)$ at Z1 and Z2 indicates an early manifestation of the phase transition from the aperiodic state to the periodic state. Figure 3 shows the evolution from aperiodic dynamics to periodic oscillations (picture insets) at Z1 (Fig. 3a), Z2 (Fig. 3b) and $\mathrm{Z} 3$ (Fig. 3c). At Z1 and Z2, $\dot{q}^{\prime}(t)$ has a low amplitude and is aperiodic before the OOD, while near the OOD, the amplitude of $\dot{q}^{\prime}(t)$ increases at both Z1 and Z2. In fact, the increase in amplitude of $\dot{q}^{\prime}(t)$ manifests as a localized emergence of periodic flame oscillations at these regions. This increase in $\dot{q}^{\prime}(t)$ is evident from the substantial increase in $\sigma^{2}$ at these locations (Fig. 3f). The high $\dot{q}^{\prime}(t)$ at 
Z2 can be attributed to the flapping of the shear layer which is one of the underlying mechanisms of $S_{T I}{ }^{63}$. The maximum value of $\sigma^{2}$ at $\mathrm{Z} 1$ and $\mathrm{Z} 2$ occurs near the OOD, indicating that they form a pair of zones wherein critical phenomena occurs first.

On the other hand, this critical phenomena is neither seen in global measures of $p^{\prime}(t)$ and $\dot{Q}^{\prime}(t)$ nor in $\sigma^{2}$ at other regions of the combustor prior to the OOD. In particular, both the $p_{r m s}^{\prime}$ (root mean square of the acoustic pressure fluctuations) and $\sigma^{2}$ of the full combustion zone show a negligible increase before the OOD (Fig. 3e-f). Hence, there is no indication of a phase transition in the global dynamics. At the same time, local flame fluctuations at Z1 and Z2 indicate an early manifestation of the transition from $S_{C}$ to $S_{T I}$. In contrast, $\sigma^{2}$ at the wake remains low for the entire duration of the experiment. The local flame fluctuations in the wake may be aperiodic or periodic with low strength of fluctuations. At Z4, the growth of $\dot{q}^{\prime}(t)$ is relatively small compared to that at Z1 and Z2 (Fig. 3a-d) but has a higher growth of $\sigma^{2}$ compared to the wake region (inset of Fig. 3f).

Even though the importance of Z3 during $S_{T I}$ due to the emergence of large-scale coherent vortices is clear, Z3 does not show the earliest indication of the phase transition. The amplitude of $\dot{q}^{\prime}(t)$ at Z3 grows gradually with increase in $\dot{m}_{\text {air }}$. In fact, $\sigma^{2}$ at Z3 closely matches the growth of $p_{\text {rms }}^{\prime}$ in comparison to $\sigma^{2}$ at Z1 and Z2 (Fig. 3e). This close match of the variation of $\sigma^{2}$ at Z3 and $p_{r m s}^{\prime}$ is expected because of the influence of impingement of large-scale coherent flow structures formed from spatial organization of small-scale vortices during $S_{T I} 9,44,64,65$. On the other hand, at $S_{C}$, such spatial organization does not occur, resulting in low amplitude aperiodic flame fluctuations. Thus, although $\dot{q}^{\prime}(t)$ at Z3 is high during $S_{T I}$, the growth in $\sigma^{2}$ is low before the OOD. However, the localized increase in periodic fluctuations of $\dot{q}^{\prime}(t)$ at Z1 and Z2 may eventually enhance the mutual feedback between $p^{\prime}(t)$ and $\dot{q}^{\prime}(\mathbf{x}, t)$, pushing the state of the system from $S_{C}$ to $S_{T I}$.

\section{Teleconnections within the flame near the phase transition.}

Correlation analysis shows that Z1, Z2 and Z3 indicate a localized increase in long-range connections or teleconnections between them before the OOD. We utilize Pearson correlation ${ }^{66}$ and Moran's spatial correlation ${ }^{67}$ to analyze the correlations within the combustion zone (More details in Methods). Spatial order emerges in the flame and flow dynamics from $S_{C}$ to $S_{T I}{ }^{44,68}$. Pearson correlation coefficient of $\dot{q}^{\prime}(t)\left(C C_{\dot{q}^{\prime}}\right)$ between Z1, Z2 and Z3 reveals non-local and long-range connections between them near the OOD (Fig. 4a). In fact, we observe that $C C_{\dot{q}^{\prime}}$ between $\mathrm{Z} 1, \mathrm{Z} 2$ and $\mathrm{Z} 3$ increases prior to the OOD. The correlation between these regions is statistically significant with $p$-value $<0.001$ (see supplementary Fig. 9). Clearly, the increase in $C C_{\dot{q}^{\prime}}$ corresponds to the increase in $\sigma^{2}$ at both $\mathrm{Z} 1$ and Z2. Such an increase in $C C_{\dot{q}^{\prime}}$, especially before any appreciable change in the global measures of $p_{r m s}^{\prime}$ and $\dot{Q}_{r m s}^{\prime}$, indicates an emerging teleconnection between Z1, Z2 and Z3 before the OOD. On 

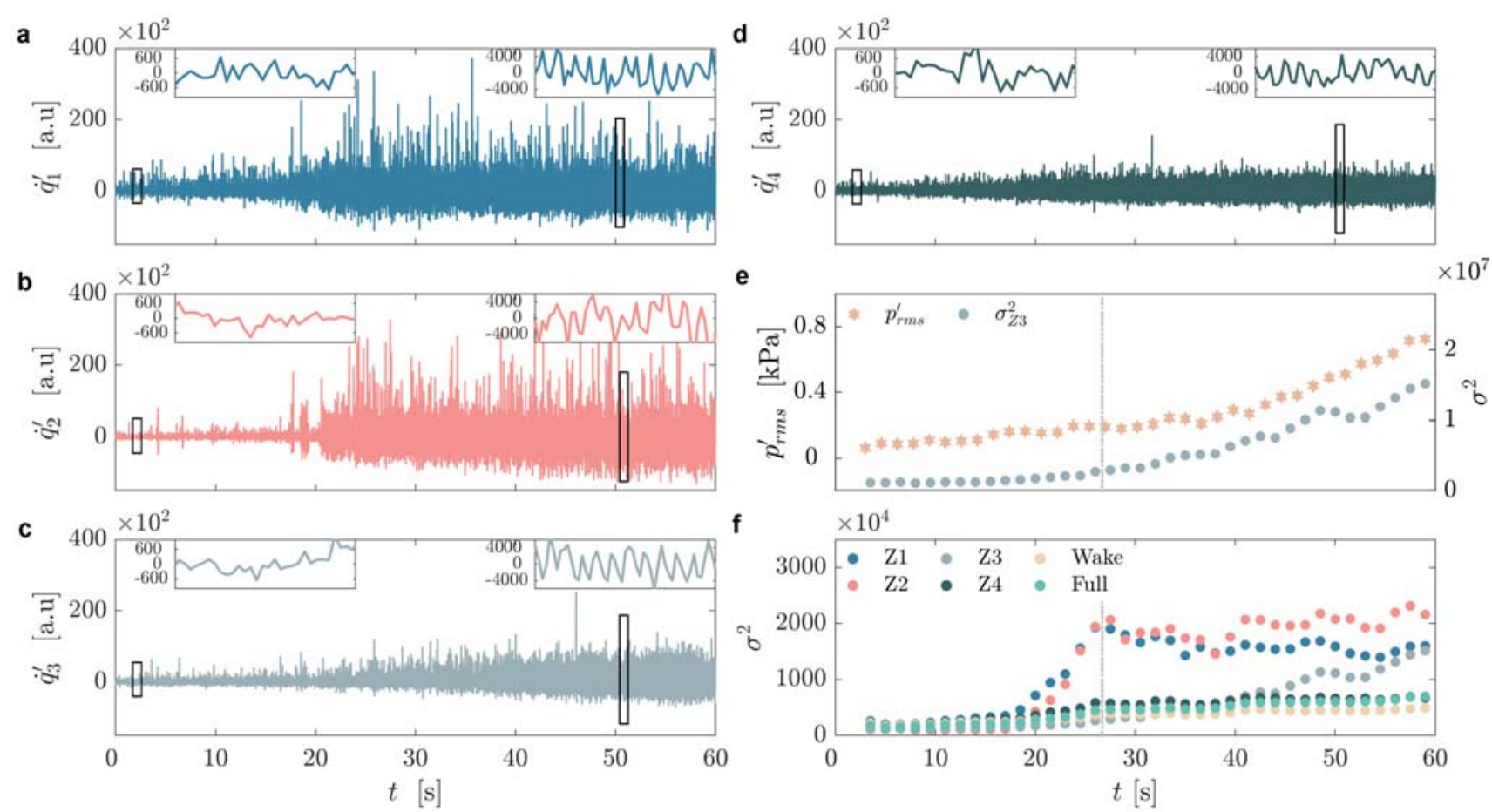

Figure 3: Critical phenomena at $\mathrm{Z1}$ and $\mathrm{Z2}$ indicate the earliest manifestation of the onset of thermoacoustic instability. The time series of $\dot{q}^{\prime}(t)$ at (a) Z1, (b) Z2, (c) Z3 and (d) Z4 show an increase in the amplitude of the fluctuations from $t=0$ to $t=60 \mathrm{~s}$. (e) The time series of $p_{r m s}^{\prime}$ shows an exponential growth from $t=4$ to $t=60$. The accuracy of the exponential fit is given by $R^{2}=0.984$. (f) The time series of $\sigma^{2}$ of $\dot{q}^{\prime}(t)$ at the Zs, the entire wake region and the full combustion zone. $\sigma^{2}$ for the full region shows little change in their value. The $\sigma^{2}$ at $\mathrm{Z} 1$ and $\mathrm{Z} 2$ increases dramatically before the OOD indicating the proximity to an imminent phase transition. This increase in $\sigma^{2}$ at these $\mathrm{Z1}$, $\mathrm{Z} 2$ and $\mathrm{Z} 3$ occurs before any visible growth in $p_{r m s}^{\prime} . \sigma^{2}$ at $\mathrm{Z} 3$ increases after the OOD and matches with the growth of $p_{r m s}^{\prime}$ as compared to $\mathrm{Z} 1$ and $\mathrm{Z} 2$. At $\mathrm{Z} 4, \sigma^{2}$ is high compared to the other regions at the beginning of the experiment and shows a linear increase prior to OOD. The OOD is denoted by a dotted vertical line at $t=26.5 \mathrm{~s}$. For the time series of $\dot{q}^{\prime}(t)$ and $\sigma^{2}$ we utilize the spatial mean of $\dot{q}^{\prime}(t)$ over certain areas: for Z1, Z2 and Z3, we utilize an area of $5 \times 5$ pixels, for Z4: $20 \times 20$ pixels, for wake: $120 \times 120$ pixels. All the measures are then normalized with respect to the area.

the contrary, beyond the OOD, the correlation between these zones decrease, when one may expect it to increase because the amplitude of the periodic oscillations continues to grow after the OOD (see Fig. 1). But, we also observe that the spatial extent of the correlated behaviour increases as the system approaches $S_{T I}$. In fact, we observe an increase in spatial correlation of the local heat release rate fluctuations $R_{\dot{q}^{\prime}}$ (Fig. 4b) with increase in $\dot{m}_{a i r}$ (More details on calculation of $R_{\dot{q}^{\prime}}$ is in Methods). $R_{\dot{q}^{\prime}}$ fluctuates around 0.34 before exhibiting a continuous increase in $R_{\dot{q}^{\prime}}$ after $t=18 \mathrm{~s}$. Thus, even 

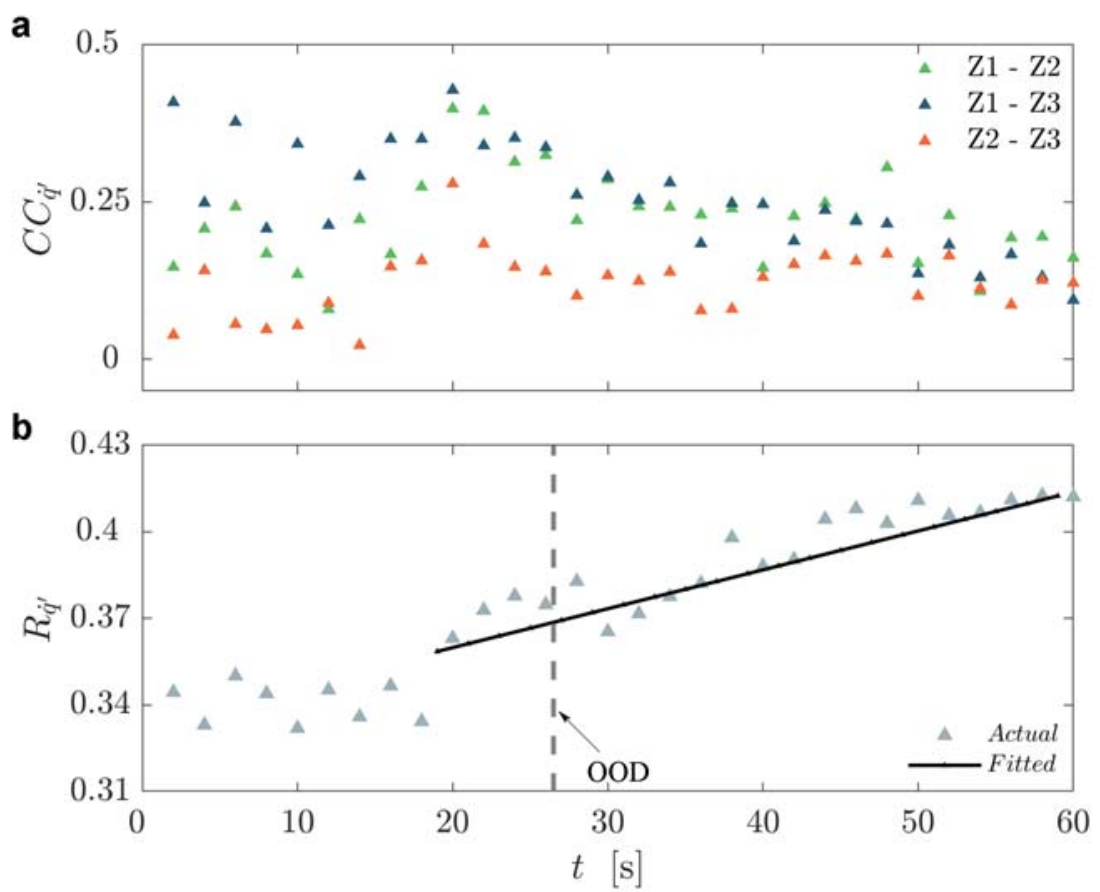

Figure 4: Emergence of interconnections prior to the transition to thermoacoustic instability. (a) Time series of Pearson correlation coefficients $\left(C C_{\dot{q}^{\prime}}\right)$ of $\dot{q}^{\prime}(t)$ between Z1, Z2 and Z3. The high value of $\left(C C_{\dot{q}^{\prime}}\right)$ between $\mathrm{Z1}, \mathrm{Z} 2$ and $\mathrm{Z3}$ just before the OOD indicates a non-local correlation (teleconnection) between $\mathrm{Z} 1, \mathrm{Z} 2$ and $\mathrm{Z} 3$. We find that the correlations between these zones are significant (see supplementary Fig. 9). We also estimate that the increase in Pearson correlation between the Z1, Z2 and Z3 prior to OOD by utilizing the Mann-Kendall test between $t=10 \mathrm{~s}$ to $t=20 \mathrm{~s}$, skipping 0.5 $\mathrm{s}$ is significant $(p$ - values $<0.001)$. We evaluate the increase in Pearson correlation in this time period because it is prior to OOD and shows simultaneous increase in correlation for all the three cases. We note that for Z1-Z3, the correlation switches between high and low values between $t=0$ and $t=10 \mathrm{~s}$. However, it is only after $t=10 \mathrm{~s}$ that it increases continuously till $t=20 \mathrm{~s}$ (b) Time series of Moran's spatial correlation of local heat release rate fluctuations $R_{\dot{q}^{\prime}}$ that exhibit the increase in spatial extent of local heat release rate fluctuations. The spatial correlation of local heat release rate increases from $S_{C}$ to $S_{T I}$. Utilizing the Mann Kendall test, we find the $p$ value obtained for the increase in spatial correlation from $t=10 \mathrm{~s}$ to $t=81 \mathrm{~s}$, skipping $2 \mathrm{~s}$ is less than 0.001 .

though after the OOD the temporal correlation between the zones Z1, Z2 and Z3 decreases, the spatial correlation in the heat release rate fluctuations within the entire flame zone increases.

\section{Identification of the critical threshold and predicting the phase transition in advance.}

Analysis of the strength of the local flame fluctuations reveal a crucial temporal relationship between Z2 and Z4. As we utilize a sliding window approach to obtain the fluctuations of the heat 
release rate, the rms of these fluctuations: $\dot{q}_{r m s}^{\prime}$ becomes equivalent to the square root of $\sigma^{2}$. Nevertheless, since the rms value has traditionally been used to define the strength of the flame fluctuations, we utilize the rms of $\dot{q}^{\prime}(\mathbf{x}, t)$ to define the relationship between Z2 and Z4. Notably, even though the utilization of $\dot{q}_{r m s}^{\prime}$ is not new, phenomena of emerging correlations or synchronization of local heat release rate fluctuations has been investigated only recently ${ }^{43,44}$. Further, $\dot{q}_{r m s}^{\prime}$ has traditionally been utilized to estimate only the mean field conditions of the system. It is only recently that $\sigma^{2}$ has been employed on temporal fluctuations to obtain early warning signals in thermoacoustic systems ${ }^{47}$. However, utilizing the increase in the rms (or $\sigma^{2}$ ) of spatial measures has never been explored to identify the phase transition nor provide early warning measures.

Figure 5a shows that $\dot{q}_{r m s}^{\prime}$ at Z2 abruptly shifts from 800 a.u at $\dot{m}_{a i r}=580 \operatorname{SLPM}(t=14 \mathrm{~s})$ to 4000 a.u at $\dot{m}_{a i r}=610 \operatorname{SLPM}(t=24 \mathrm{~s})$, indicating a high magnitude transition. Such a high magnitude transition is a key feature of a phase transition from one state to another state. Further, as seen earlier in Fig. 2d, the earliest manifestation of critical phenomena also occurs at Z1 and Z2 wherein $\mathrm{Z} 2$ shows the highest growth in $\sigma^{2}$ of the local flame fluctuations. These features of the phase transition at Z2 makes it an ideal candidate to demarcate the chaotic state $\left(S_{C}\right)$ and the periodic state $\left(S_{T I}\right)$. On the other hand, $\dot{q}_{r m s}^{\prime}$ at Z4 is slightly higher than at Z2 during the state of $S_{C}$ and increases with $\dot{m}_{a i r} . \dot{q}_{r m s}^{\prime}$ at Z4 and Z2 intersect before OOD near the mid point between the low values and high values of $\dot{q}_{r m s}^{\prime}$ at Z2. This intersection of $\dot{q}_{r m s}^{\prime}$ at Z2 and Z4 means an equalization of the strength of local flame fluctuations at Z2 and Z4 and it occurs for all the seven experiments prior to the OOD. For the experimental data shown in Fig. 5a, the intersection occurs at $\dot{m}_{a i r}=597$ SLPM, where $\dot{q}_{r m s}^{\prime}=1839$ a.u. We denote $\dot{q}_{r m s}^{\prime}$ at the point of intersection as $\dot{q}_{r m s}^{\prime i}$. Indeed, there are some fluctuations near the intersection because of the turbulent flow field, which pushes the local dynamics back and forth between the chaotic state (low $\dot{q}_{r m s}^{\prime}$ ) and large amplitude periodic state (high $\dot{q}_{r m s}^{\prime}$ ) due to its inherent stochasticity. Nevertheless, we consider the last intersection to denote $\dot{q}_{r m s}^{\prime i}$.

Next, utilizing the point of intersection $\dot{q}_{r m s}^{\prime i}$, we define the critical threshold. We select four out of the seven experiments to make a training set. We choose the median of $\dot{q}_{r m s}^{\prime i}$ from these four experiments to define a critical threshold: $\dot{q}_{r m s}^{\prime c}=1555$ a.u. We construct this definition of the critical threshold because once the strength of the local flame fluctuations at Z2 increases beyond $\dot{q}_{r m s}^{\prime c}$, these fluctuations do not return to their low amplitude chaotic state but continue to grow in amplitude and periodicity enroute to $S_{T I}$ for all the experiments. Nair and Sujith ${ }^{46}$ proposed a user-defined threshold of 0.1 for the Hurst exponent $(H)$ of $p^{\prime}(t)$ to demarcate the state of $S_{T I}$ from $S_{C}$. Notably, $H$ decreases to 0.1 at the intersection point of Z2 and Z4 (see supplementary Fig. 10b). This feature further substantiates our view that the occurrence of the critical phenomena at Z2 and Z1 locally indicates the onset of thermoacoustic instability. 


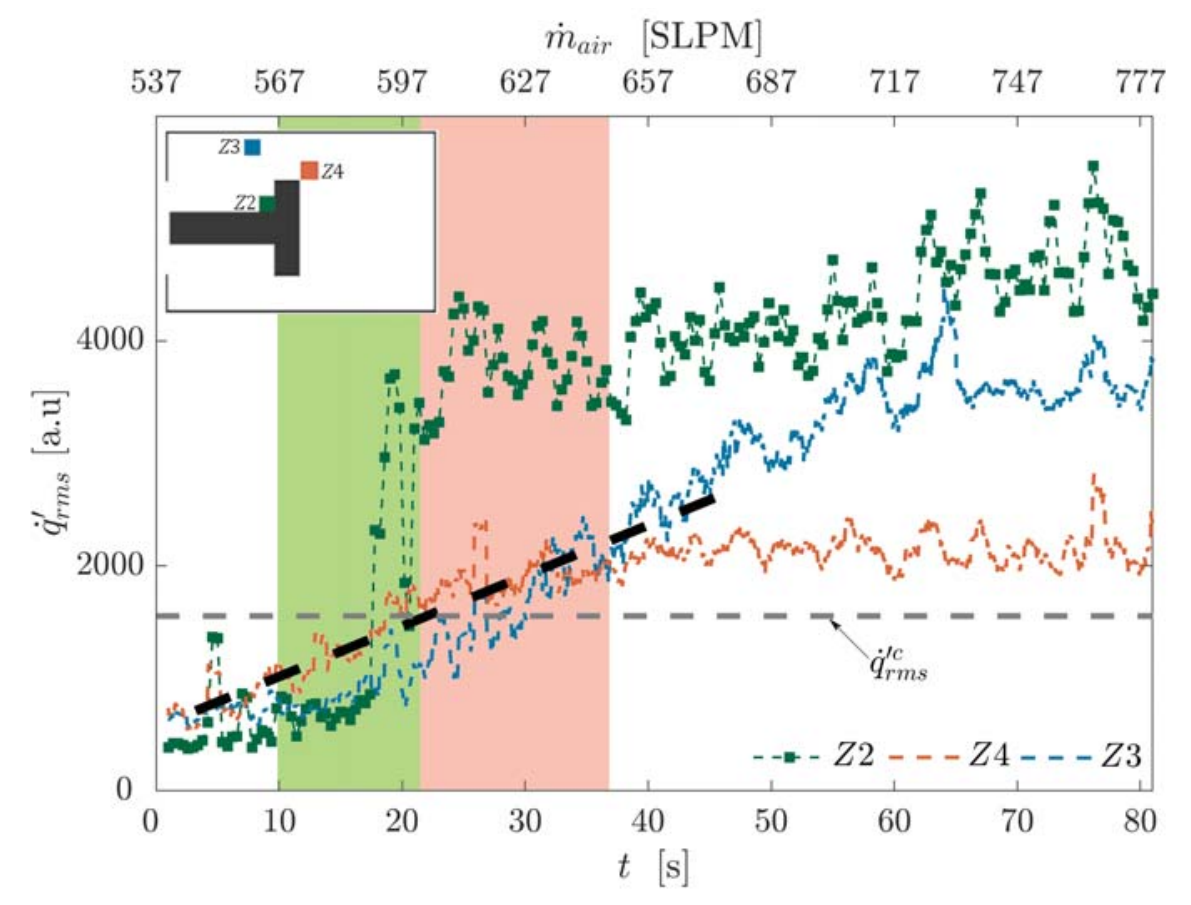

Figure 5: Increase in $\dot{q}_{r m s}^{\prime}$ at the interconnected zones mark the phase transition. This figure shows the variation of strength of the local flame fluctuations indicated by the root mean square of the heat release rate fluctuations $\dot{q}_{r m s}^{\prime}$ with respect to mass flow rate of air and time at Z2, Z3 and Z4. $\dot{q}_{r m s}^{\prime}$ at Z1 is similar to that at Z2 and is shown in supplementary Fig. 10a. $\dot{q}_{r m s}^{\prime}$ at Z2 and Z4 intersect before the OOD. The horizontal dashed line represents the median of the $\dot{q}_{r m s}^{\prime i}$ corresponding to the critical threshold for four experiments $(\mathrm{i}=1,2,3,4)$. This median value $\dot{q}_{r m s}^{\prime c}$ is 1555 a.u (arbitrary units). The green band indicates the prediction horizon to the phase transition. The right border of the pink band indicates when $\dot{q}_{r m s}^{\prime}$ at $\mathrm{Z3}$ and Z4 intersect. The inset represents the sketch of the turbulent combustor and the zones with respect to the bluff body.

Subsequently, we utilize this $\dot{q}_{r m s}^{\prime c}$ (marked by the grey dashed line in figure 5a) to predict the onset in the other three experiments. At Z4, the $\dot{q}_{r m s}^{\prime}$ increases linearly till the OOD that may be attributed to the gradual increase in the frequency of vortex shedding from the tip of the bluff body depending on $\dot{m}_{\text {air }}$. We attempt to predict the onset by making a linear fit of $\dot{q}_{r m s}^{\prime}$ at Z4 for each of the three experiments and identify where the linear fit intersects $\dot{q}_{r m s}^{\prime c}$ (see dashed black line in Fig. 5a). Table 1 shows the results from the prediction. The second column of Table 1 represents the mass flow rate of air $\dot{m}_{a}$ where the intersection between $\dot{q}_{r m s}^{\prime}$ at Z2 and Z4 occur. The third column shows the predicted air mass flow rate $\left(\dot{m}_{p}\right)$ obtained from the linear fit and $\dot{q}_{r m s}^{\prime c}$. The discrepancy between $\dot{m}_{a}$ and $\dot{m}_{p}$ is within $1 \%$, for the three test cases. These discrepancies are within the uncertainty limits of the mass flow controller. In general, this methodology predicts the earliest manifestation of the onset 11 seconds in advance, which corresponds to a change of $\dot{m}_{\text {air }}=33$ SLPM. 
The choice of the reference zone Z4 to define the critical point and predict the phase transition is not arbitrary. Unlike the interconnected zones, the $\dot{q}_{r m s}^{\prime}$ at Z4 increases from the beginning of the experiment. Once the phase transition occurs, $\dot{q}_{r m s}^{\prime}$ at Z4 saturates to a value close to 2000 a.u. On the other hand, at the wake, the $\dot{q}_{r m s}^{\prime}$ always remains low while regions within the area spanned by Z1 and Z2 show a phase transition near the OOD (not shown here). Meanwhile, at regions near Z3, the large magnitude phase transition occurs slower, as indicated by the slow increase in $\dot{q}_{r m s}^{\prime}$ at Z3. Hence, Z4, is a special location, representing a boundary between the zones wherein the the earliest manifestation of phase transition occurs (Z1 and Z2) and where the phase transition ceases to exist (wake).

Interestingly, $\dot{q}_{r m s}^{\prime}$ at Z3 and Z4 also intersect near the mid point between the low values and high values of $\dot{q}_{r m s}^{\prime}$ at Z3. This intersection occurs at $m_{a}=645$ SLPM, marked by the right border of the vertical pink band. We observe that at this point, the amplitude of the pressure oscillations reach 25 $\%$ of the maximum observed amplitude, giving us an insight to how early the onset is. In fact, this intersection occurs $16 \mathrm{~s}$ later than the earliest manifestation of the phase transition at Z1 and Z2. Z3 shows high $\dot{q}_{r m s}^{\prime}$ when large-scale coherent flow structures emerge. This shows clear evidence that the earliest manifestation of the phase transition occurs in advance to the emergence of large-scale coherent structures. Such features of Z4 and its relationship with Z2 motivates its use in defining the onset of thermoacoustic instability and predicting it.

We hypothesize that the features at Z1, Z2, Z3 and Z4 would remain the same irrespective of the changes in the experimental conditions such as different fuel flow rates, different rate of change of mass flow rate of air. The features persist due to following reasons. Firstly, at Z1, which is just above the bluff body, high heat release rate occurs after impingement of the large-scale flow structures ${ }^{44}$. Secondly, at Z3, which is located upstream of the bluff body, large-scale flow structures emerging during the state of thermoacoustic instability is ubiquitous and has been reported in multiple studies in the same experimental setup ${ }^{44,45}$. Finally, the divergent flow at Z2 (corner of bluff body and shaft) and the vortex shedding at Z4 (between the wake and Z1) are imposed by the geometry of the bluff body. Hence, we expect critical phenomena to occur locally at Z1 and Z2 before the phase transition, irrespective of changes in experimental conditions.

\section{Discussion}

We have presented comprehensive experimental evidence on identifying two interconnected zones seeds of the phase transition in a turbulent reactive flow system that point to the earliest manifestation of the phase transition. First, we have unravelled those zones, where the earliest manifestation of 


\begin{tabular}{cccc} 
S1.No & $\dot{m}_{a}$ & $\dot{m}_{p}$ & $\delta(\%)$ \\
\hline \hline 1 & 587 & 585 & 0.34 \\
2 & 596 & 590 & 1.00 \\
3 & 599 & 601 & -0.33 \\
\hline
\end{tabular}

Table 1: Utilizing the interconnected zones show high accuracy in predicting the phase transition. Key: $\dot{m}_{a}$ - Mass flow rate of air at which $\dot{q}_{r m s}^{\prime}$ of Z2 crosses Z4 for the experiment; $\dot{m}_{p}$ - Predicated value of air mass flow rate in SLPM utilizing the regression line from the time series at Z4 and the critical threshold $\dot{q}_{r m s}^{\prime c} ; \delta$ - Standard error in percentage and is given as $\left(\dot{m}_{a}-\dot{m}_{p}\right) / \dot{m}_{a}$. The discrepancy between $\dot{m}_{a}$ and $\dot{m}_{p}$ is within the uncertainty limits of the controller at these flow rates ( \pm 9 SLPM).

the onset of phase transition appears, utilizing the increase in the variance of local fluctuations of the flame. Second, the emergence of long-range correlations between these zones prior to the onset of the phase transition has been found. Next, we have identified the critical threshold of the flame fluctuations at these interconnected zones. These findings enable us to identify the onset of a phase transition from a chaotic state to a periodic state, which in our case is the onset of thermoacoustic instability. Notably, all precursors which have been shown in recent decades are indicators of an impending thermoacoustic instability. To the best of the authors' knowledge, a prediction of when the onset will occur has not been shown in any experimental, numerical or analytical study to date. Our result opens promising perspectives for predicting the onset of transitions in complex systems wherein acquisition of spatiotemporal data is possible.

Previously, Stolbova et al. ${ }^{29}$ revealed tipping elements in the Indian monsoon system by analyzing observational data of atmospheric variables, utilizing the time series at the tipping elements. These tipping elements allowed predicting the onset of the monsoon with large lead times. We have adapted the tipping elements approach for turbulent combustor and have developed it further for the case of thermoacoustic instability. This methodology allows to predict the earliest manifestation of the onset before it appears. Importantly, the methodology that we have used to identify the zones where the earliest manifestation of the onset appears do not need detailed information of the influential processes that drive the transition. In fact, the earliest manifestation of the phase transition is found only by utilizing the criterion, which is the growth in the variance of fluctuations of certain system variables. The evidence from both observational data analysis and our experimental results shows the applicability of the methodology for various complex systems and demonstrate the predictive power with sufficient lead times. The methodology requires only spatiotemporal information of system 
variables at different conditions of a control parameter that allows to identify critical phenomena prior to the phase transition.

The suppression of the onset of the phase transition may open new perspectives for "greener" operations. We hypothesize that the interconnected zones perform as bridges to ordered dynamics that initiate positive feedbacks within the system, necessary for the phase transition to occur. Suppressing the spatiotemporal organization in such zones should break a pathway to $S_{T I}$. In general, most control strategies intend to destroy large-scale coherent flow structures that exist at $S_{T I}$. However, we have revealed that the onset of $S_{T I}$ at the interconnected zones $\mathrm{Z} 1$ and $\mathrm{Z} 2$ occurs even before the emergence of these large-scale coherent structures. Our findings suggest that it may be necessary and useful to implement control strategies at this onset of $S_{T I}$ which appears much before the large amplitude oscillations of $p^{\prime}$ occur. The revealed interconnections between these zones suggests a promising way to suppress the phase transition by disrupting the linkage between them and thus block a pathway to $S_{T I}$. Further, the question of whether suppressing critical phenomena at the interconnected zones can suppress thermoacoustic instability also remains an open question. Pursuing this research question is important for developing passive control strategies. Preliminary experiments show that suppression of thermoacoustic instability close to $90 \%$ is possible by adopting such a strategy, but this is for future study. Thus, our study provides a very-much-needed understanding of experimental evidence showing the special role of the seeds of phase transition ${ }^{69}$ that mark the earliest manifestation of the transition to thermoacoustic instability, which allow us to define the onset of thermoacoustic instability. This is a crucial step for "greener" operations.

\section{Methods}

Experimental setup. We perform experiments in a turbulent thermoacoustic system. The turbulent combustor consists mainly of a plenum chamber, a burner and a combustion chamber. The schematic of the experimental setup is shown in Fig. 6. The flame stabilizes inside the combustor on a flame holding device called a bluff-body shown as an inset. Air and Liquefied petroleum gas (LPG: butane $60 \%$ and propane 40\%) mix just before the burner. Most of the reaction occurs in the combustion chamber. In this study, we vary the fuel-air mixture ratio to control the system characteristics. Mass flow controllers (Alicat Scientific, MCR Series) are used to control and measure the mass flow rates of air and fuel, with an uncertainty of $\pm 0.8 \%$ of the flow rate reading in SLPM $+0.2 \%$ of the maximum flow rate of the air flow controller: 2000 SLPM. The fuel-air mixture ratio is varied linearly by increasing the air mass flow rate at a rate of 3 SLPM (with uncertainty \pm 0.11 SLPM) per second while keeping the fuel mass flow rate fixed at 34 SLPM.

We acquire $p^{\prime}(t)$ using a piezoelectric pressure transducer (PCB103B02, uncertainty $\pm 0.15 \mathrm{~Pa}$ ). 


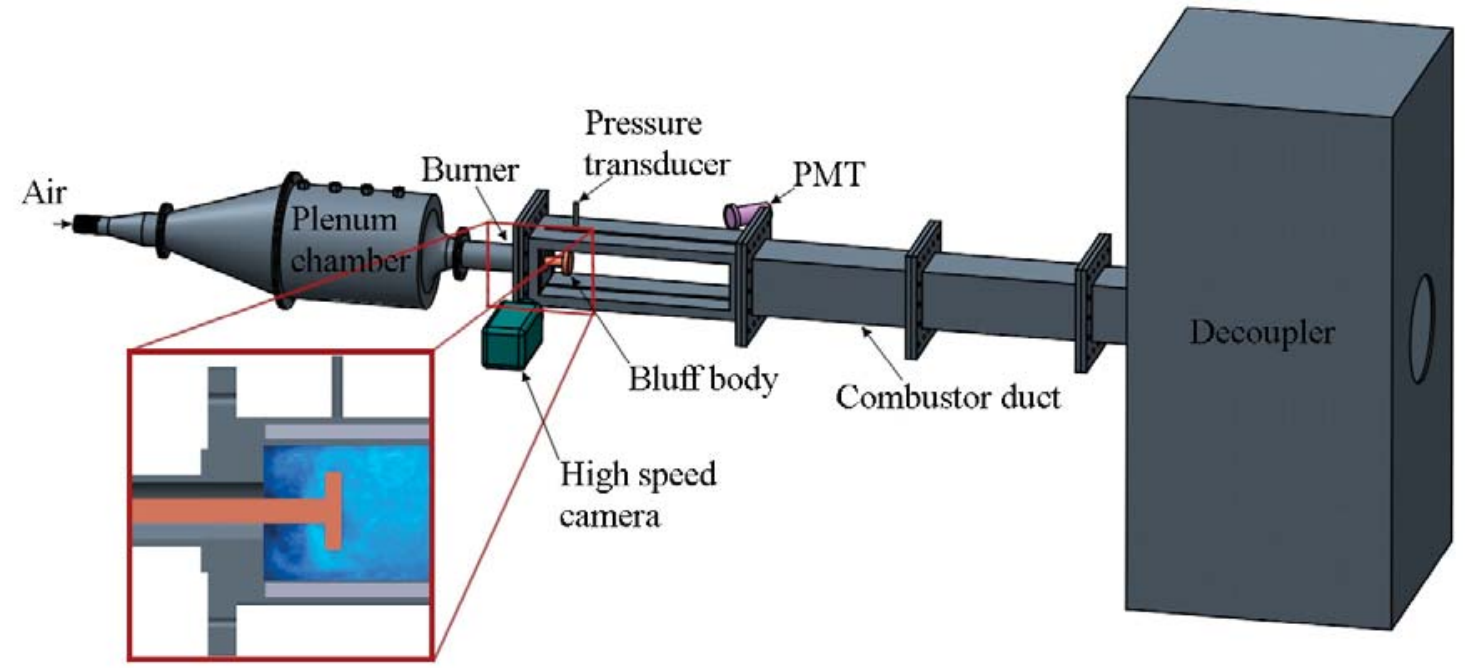

Figure 6: Schematic of the experimental setup. (a) Bluff-body stabilized turbulent thermoacoustic instability system. We simultaneously measure acoustic pressure fluctuations $p^{\prime}(t)$, global heat heat release rate $\dot{Q}^{\prime}(t)$, and high speed $C H^{*}$ chemiluminescence field as a function of fuel-air mixture ratio. The inset shows the zoomed version of the combustion chamber with the bluff body that is used to stabilize the flame.

In order to record the maximum amplitude of the standing wave, the sensor is mounted at the antinode of the acoustic oscillations ( $25 \mathrm{~mm}$ distance from the backward-facing step of the combustor). The local $\mathrm{CH}^{*}$ chemiluminescence intensity represents $\dot{q}(\mathbf{x}, t)$ within the combustor. The global $\mathrm{CH}^{*}$ chemiluminescence intensity $\dot{Q}(t)$ is captured using a photomultiplier (PMT, Hamamatsu H10722$01)$ mounted with a $C H^{*}$ filter $\left(435 \mathrm{~nm} \pm 12 \mathrm{~nm}\right.$ FWHM) in front of it. Both $p^{\prime}(t)$ and $\dot{Q}(t)$ are acquired at a sampling rate of $10 \mathrm{kHz}$ using an analogue-to-digital card (NI-6143, 16 bit). The spatial chemiluminescence intensity $(\dot{q}(\mathbf{x}, t))$ is captured using a Phantom V12.1 high-speed camera (of spatial resolution $800 \times 600$ pixels) equipped with a Zeiss $100 \mathrm{~mm}$ lens and a $\mathrm{CH}^{*}$ filter. The images are captured at a sampling rate of 500 frames per second for a duration of $84 \mathrm{~s}$. All these measurements are acquired simultaneously. Detailed descriptions of the experimental configuration, data recording systems and control systems can be found in George et $a l^{44}$.

\section{Estimating the composite averages of $\sigma^{2}$ field based on identifying the onset of ordered dynamics (OOD)}

We analyze the phase difference between $p^{\prime}(t)$ and $\dot{Q}^{\prime}(t)$ to estimate a common reference between the multiple experiments since the individual global measures do not reveal a period wherein the transition occurs. We calculate the instantaneous phase $(\delta \theta)$ between $p^{\prime}(t)$ and $\dot{Q}^{\prime}(t)$ using Hilbert transform $(\mathrm{HT})^{70}$. At first, we compute the phase and amplitude of $p^{\prime}(t)$ and $\dot{Q}^{\prime}(t)$ individually us- 
ing HT. Then the relative phase between $p^{\prime}(t)$ and $\dot{Q}^{\prime}(t)$ is calculated as the difference between the computed phase of those signals. In general, the relative phase between two signals is bounded in an interval [-180 180]. However, here, we unwrapped $\delta \theta$ obtained from HT to visualize how $\delta \theta$ continuously changes during the transition from combustion noise to thermoacoustic instability.

a

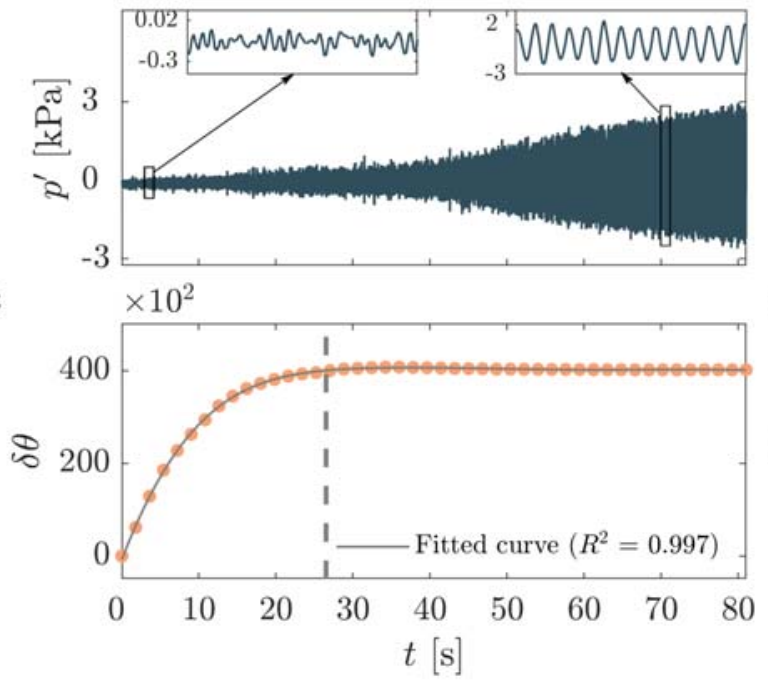

b

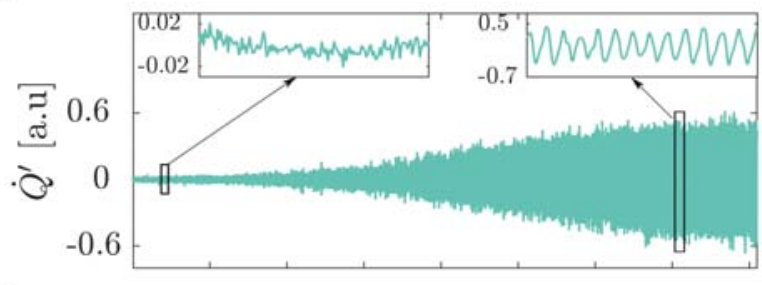

d

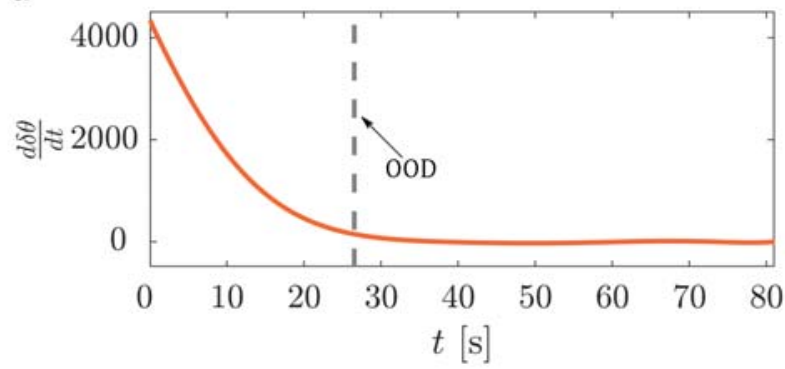

Figure 7: Transition from low amplitude chaotic fluctuations to high amplitude periodic oscillations in a turbulent thermoacoustic system. The time series of (a) $p^{\prime}(t),(\mathbf{b}) \dot{Q}^{\prime}(t)$, (c) the phase difference between $p^{\prime}(t)$ and $\dot{Q}^{\prime}(t): \delta \theta$, (d) first derivative of phase difference: $\frac{d \delta \theta}{d t}$, calculated using first order finite difference method. All time series are shown for continuous variation of $\dot{m}_{\text {air }}$ from $537 \mathrm{SLPM}$ at $t=0$ to $780 \mathrm{SLPM}$ at $t=81 \mathrm{~s}$. The insets in $\mathbf{a}$ and $\mathbf{b}$ show zoomed-in views of $p^{\prime}(t)$ and $\dot{Q}^{\prime}(t)$ during $S_{C}$ and $S_{T I}$. The unwrapped phase difference $\delta \theta$ increases till $t=25 \mathrm{~s}$ and becomes constant. Utilizing a near zero value of $\frac{d \delta \theta}{d t}$, we mark the point of the onset of ordered dynamics (OOD) by a dashed vertical gray line. At the OOD, $\dot{m}_{a i r}$ and $R e$ are 617 SLPM and 16380 respectively.

Figure 7c shows the continuously changing phase difference $(\delta \theta)$ from $t=0 \mathrm{~s}$, which begins to converge to an asymptotic value near $t=25 \mathrm{~s}$. Previous studies have shown that as the $p^{\prime}(t)$ and $\dot{Q}^{\prime}(t)$ become periodic, their phase difference becomes constant ${ }^{71}$. Next, we calculate the first derivative of $\delta \theta: \frac{d \delta \theta}{d t}$ (Fig. 7d). As the phase difference becomes constant, the first derivative of the phase difference will approach zero. We mark the time instant wherein the $\frac{d \delta \theta}{d t}$ reaches $99.99 \%$ of the difference between the largest positive value and zero, and refer to it as the onset of ordered dynamics (OOD). For experimental data shown in Fig. 7, we identify the OOD at $t=26.5 \mathrm{~s}$ where $\dot{m}_{\text {air }}$ is 617 SLPM. The same approach is followed to obtain OOD for the other experiments. Subsequently, we estimate averages across the seven experiments with respect to OOD as the reference point from each experiment. These averages, referred to as composite averages are shown in Fig. 2 for $\sigma^{2}$ fields at 
different instants of time with respect to OOD. We utilize these composite averages to standardize $\sigma^{2}$ prior to the OOD.

Calculation of variance of fluctuations of local heat release rate. We calculate the variance of fluctuations $\sigma^{2}$ of $\dot{q}(\mathbf{x}, t)$ at each pixel for a time period of $w=3.5 \mathrm{~s}$ wherein the fluctuations are calculated based on a moving average of $w_{s}=0.04 \mathrm{~s} . \quad d$ refers to the time instant at which the calculation is performed.

$$
\sigma^{2}\left(\dot{q}, d, w, w_{s}\right)=\sum_{k=1}^{w} \frac{\left[\dot{q}(O O D-d-k)-\sum_{i=1}^{w_{s}} \frac{\dot{q}(O O D-d-k-i)}{w_{s}}\right]^{2}}{w}
$$

For example, assuming OOD is at $25 \mathrm{~s}$, to calculate $\sigma^{2} 20 \mathrm{~s}(d)$ before the OOD, we utilize the fluctuations from $t=1.5 \mathrm{~s}$ to $t=5 \mathrm{~s}$.

Pearson correlation coefficients of local heat release rate fluctuations at $\mathbf{Z 1}, \mathbf{Z 2}$ and $\mathbf{Z 3}$. We compute the correlation coefficients $C C_{\dot{q}^{\prime}}$ of $\dot{q}^{\prime}(t)$ between various zones. We calculate the Pearson correlation coefficient for every window as

$$
C C_{\dot{q}^{\prime}}=\frac{1}{N-1} \sum_{i=1}^{N}\left(\frac{\dot{a}_{i}^{\prime}}{\sigma_{\dot{a}^{\prime}}}\right)\left(\frac{\dot{b}_{i}^{\prime}}{\sigma_{\dot{b}^{\prime}}}\right)
$$

where $\dot{a}^{\prime}$ and $\dot{b}^{\prime}$ are the time series of $\dot{q}^{\prime}$ at different zones (for example Z1 and Z2), $\sigma$ is the standard deviation of the respective time series. Here we used a window size $(N)$ of 4 s to compute $C C_{\dot{q}^{\prime}}$. The fluctuation $\dot{q}^{\prime}(t)$ is calculated by subtracting the instantaneous quantity from a moving average of $0.04 \mathrm{~s}$.

Spatial correlation of local heat release rate fluctuations. We calculate the spatial correlation utilizing Moran's correlation ${ }^{67}$ given by

$$
R_{\dot{q}^{\prime}}=\frac{K}{\sum_{i=1}^{l} \sum_{j=1}^{m} w_{i j}} \frac{\sum_{i=1}^{l} \sum_{j=1}^{m} w_{i j}\left(X_{i}-\bar{X}\right)\left(X_{j}-\bar{X}\right)}{\sum_{i=1}^{l}\left(X_{i}-\bar{X}\right)^{2}}
$$

where $X$ is the value of $\dot{q}^{\prime}(\mathbf{x}, t)$ at different spatial locations indexed by $l$ and $m$. $K$ is given by $K=l \times m . \bar{X}$ is the spatial average of $X$ and $w_{i j}$ is a component of a matrix of spatial weights. Here, we have considered $w_{i j}$ as 1 . 


\section{References}

${ }^{1}$ Biggs, R. Rocketdyne-F-1 Saturn V first stage engine. In Remembering the Giants: Apollo Rocket Propulsion Development, 15-26 (NASA, 2009).

${ }^{2}$ Blomshield, F., Mathes, H., Crump, J., Beiter, C. \& Beckstead, M. Nonlinear stability testing of full-scale tactical motors. J. Propul. Power 13, 356-366 (1997).

${ }^{3}$ Sutton, G. P. History of liquid-propellant rocket engines in Russia, formerly the Soviet Union. $J$. Propul. Power 19, 1008-1037 (2003).

${ }^{4}$ Lieuwen, T. C. \& Yang, V. Combustion Instabilities: Basic Concepts, 3-26 (American Institute of Aeronautics and Astronautics, 2005).

${ }^{5}$ McManus, K. R., Poinsot, T. \& Candel, S. M. A review of active control of combustion instabilities. Progress. Eng. Comb. Science 19, 1-29 (1993).

${ }^{6}$ Lieuwen, T. C. Experimental investigation of limit-cycle oscillations in an unstable gas turbine combustor. J. Propul. Power 18, 61-67 (2002).

${ }^{7}$ Ducruix, S., Schuller, T., Durox, D. \& Candel, S. Combustion dynamics and instabilities: Elementary coupling and driving mechanisms. J. Propul. Power 19, 722-734 (2003).

${ }^{8}$ Juniper, M. P. \& Sujith, R. I. Sensitivity and nonlinearity of thermoacoustic oscillations. Annu. Rev. Fluid Mech. 50, 661-689 (2018).

${ }^{9}$ Poinsot, T. J., Trouve, A. C., Veynante, D. P., Candel, S. M. \& Esposito, E. J. Vortex-driven acoustically coupled combustion instabilities. J. Fluid. Mech. 177, 265-292 (1987).

${ }^{10}$ Schadow, K. et al. Large-scale coherent structures as drivers of combustion instability. Comb. Sci. Technol. 64, 167-186 (1989).

${ }^{11}$ Kline, S. J., Reynolds, W. C., Schraub, F. \& Runstadler, P. The structure of turbulent boundary layers. J. Fluid. Mech. 30, 741-773 (1967).

${ }^{12}$ Brown, G. L. \& Roshko, A. On density effects and large structure in turbulent mixing layers. $J$. Fluid. Mech. 64, 775-816 (1974).

${ }^{13}$ Cantwell, B. J. Organized motion in turbulent flow. Annu. Rev. Fluid Mech. 13, 457-515 (1981).

${ }^{14}$ Bernal, L. \& Roshko, A. Streamwise vortex structure in plane mixing layers. J. Fluid. Mech. 170, 499-525 (1986). 
${ }^{15}$ Robinson, S. K. Coherent motions in the turbulent boundary layer. Annu. Rev. Fluid Mech. 23, 601-639 (1991).

${ }^{16}$ Ball, P. \& Borley, N. R. The self-made tapestry: pattern formation in nature (Oxford University Press Oxford, 1999).

${ }^{17}$ Meron, E. Pattern formation - a missing link in the study of ecosystem response to environmental changes. Math. Biosci. 271, 1-18 (2016).

${ }^{18}$ Rastogi, B. et al. Spatial and temporal patterns of cloud cover and fog inundation in coastal california: ecological implications. Earth Interactions 20, 1-19 (2016).

${ }^{19}$ Chang, W., Stein, M. L., Wang, J., Kotamarthi, V. R. \& Moyer, E. J. Changes in spatiotemporal precipitation patterns in changing climate conditions. J. Climate 29, 8355-8376 (2016).

${ }^{20}$ Busse, F. Non-linear properties of thermal convection. Reports on Prog. Phys. 41, 1929-1967 (1978).

${ }^{21}$ Croquette, V. Convective pattern dynamics at low Prandtl number: Part I. Contemporary Physics 30, 113-133 (1989).

${ }^{22}$ Croquette, V. Convective pattern dynamics at low Prandtl number: Part II. Contemporary Physics 30, 153-171 (1989).

${ }^{23}$ Cross, M. C. \& Hohenberg, P. C. Pattern formation outside of equilibrium. Rev. Mod. Phys. 65, 851-1112 (1993).

${ }^{24}$ Camazine, S. et al. Self-organization in biological systems (Princeton University press, 2003).

${ }^{25}$ Bayliss, A. \& Matkowsky, B. J. Bifurcation, pattern formation and chaos in combustion. In $D y$ namical Issues in Combustion Theory, 1-35 (Springer, 1991).

${ }^{26}$ Scheffer, M., Carpenter, S., Foley, J. A., Folke, C. \& Walker, B. Catastrophic shifts in ecosystems. Nature 413, 591-596 (2001).

${ }^{27}$ Dakos, V. et al. Methods for detecting early warnings of critical transitions in time series illustrated using simulated ecological data. PLoS one 7, e41010 (2012).

${ }^{28}$ Lade, S. J. \& Gross, T. Early warning signals for critical transitions: A generalized modeling approach. PLoS. Comput. Biol. 8, e1002360 (2012). 
${ }^{29}$ Stolbova, V., Surovyatkina, E., Bookhagen, B. \& Kurths, J. Tipping elements of the Indian monsoon: Prediction of onset and withdrawal. Geophy. Res. Lett. 43, 3982-3990 (2016).

${ }^{30}$ Rocha, J. C., Peterson, G., Bodin, Ö. \& Levin, S. Cascading regime shifts within and across scales. Science 362, 1379-1383 (2018).

${ }^{31}$ Surovyatkina, E. Rise and saturation of the correlation time near bifurcation threshold. Phys. Lett. A 329, 169-172 (2004).

${ }^{32}$ Surovyatkina, E., Kravtsov, Y. A. \& Kurths, J. Fluctuation growth and saturation in nonlinear oscillators on the threshold of bifurcation of spontaneous symmetry breaking. Phys. Rev. E. 72, 046125 (2005).

${ }^{33}$ Rayleigh, J. W. S. The explanation of certain acoustical phenomena. Nature 18, 319-321 (1878).

${ }^{34}$ Candel, S. M. Combustion instabilities coupled by pressure waves and their active control. In Symposium (International) on Combustion, vol. 24, 1277-1296 (Elsevier, 1992).

${ }^{35}$ Lieuwen, T. C. Unsteady combustor physics (Cambridge University Press, 2012).

${ }^{36}$ Sujith, R. I. \& Unni, V. R. Complex system approach to investigate and mitigate thermoacoustic instability in turbulent combustors. Phys. Fluids. 32, 061401 (2020).

${ }^{37}$ Sujith, R. I. \& Unni, V. R. Dynamical systems and complex systems theory to study unsteady combustion. Proc. Comb. Inst. (2020).

${ }^{38}$ Gotoda, H., Nikimoto, H., Miyano, T. \& Tachibana, S. Dynamic properties of combustion instability in a lean premixed gas-turbine combustor. Chaos 21, 013124 (2011).

${ }^{39}$ Nair, V., Thampi, G. \& Sujith, R. I. Intermittency route to thermoacoustic instability in turbulent combustors. J. Fluid. Mech. 756, 470-487 (2014).

${ }^{40}$ Palies, P., Ilak, M. \& Cheng, R. Transient and limit cycle combustion dynamics analysis of turbulent premixed swirling flames. J. Fluid. Mech. 830, 681-707 (2017).

${ }^{41}$ Nair, V., Thampi, G., Karuppusamy, S., Gopalan, S. \& Sujith, R. I. Loss of chaos in combustion noise as a precursor of impending combustion instability. Int. J. Spray. Comb. Dyn. 5, 273-290 (2013).

${ }^{42}$ Tony, J., Gopalakrishnan, E., Sreelekha, E. \& Sujith, R. Detecting deterministic nature of pressure measurements from a turbulent combustor. Phys. Rev. E 92, 062902 (2015). 
${ }^{43}$ Mondal, S., Unni, V. R. \& Sujith, R. I. Onset of thermoacoustic instability in turbulent combustors: an emergence of synchronized periodicity through formation of chimera-like states. J. Fluid. Mech. 811, 659-681 (2017).

${ }^{44}$ George, N. B., Unni, V. R., Raghunathan, M. \& Sujith, R. I. Pattern formation during transition from combustion noise to thermoacoustic instability via intermittency. J. Fluid. Mech. 849, 615644 (2018).

${ }^{45}$ Raghunathan, M. et al. Multifractal analysis of flame dynamics during transition to thermoacoustic instability in a turbulent combustor. J. Fluid. Mech. 888 (2020).

${ }^{46}$ Nair, V. \& Sujith, R. I. Multifractality in combustion noise: predicting an impending combustion instability. J. Fluid. Mech. 747, 635-655 (2014).

${ }^{47}$ Gopalakrishnan, E. A., Sharma, Y., John, T., Dutta, P. S. \& Sujith, R. I. Early warning signals for critical transitions in a thermoacoustic system. Sci. Rep. 6, 1-10 (2016).

${ }^{48}$ Gotoda, H., Shinoda, Y., Kobayashi, M., Okuno, Y. \& Tachibana, S. Detection and control of combustion instability based on the concept of dynamical system theory. Phys. Rev. E 89, 022910 (2014).

${ }^{49}$ Godavarthi, V., Unni, V. R., Gopalakrishnan, E. A. \& Sujith, R. I. Recurrence networks to study dynamical transitions in a turbulent combustor. Chaos 27, 063113 (2017).

${ }^{50}$ Xiong, S., Mondal, S. \& Ray, A. Detection of thermoacoustic instabilities via nonparametric bayesian markov modeling of time-series data. J. Dyn. Sys. Meas. Cont. 140 (2018).

${ }^{51}$ Mondal, S., Ghalyan, N. F., Ray, A. \& Mukhopadhyay, A. Early detection of thermoacoustic instabilities using hidden markov models. Comb. Sci. Technol. (2018).

${ }^{52}$ Kobayashi, T., Murayama, S., Hachijo, T. \& Gotoda, H. Early detection of thermoacoustic combustion instability using a methodology combining complex networks and machine learning. Phys. Rev. App. 11, 064034 (2019).

${ }^{53}$ Hachijo, T., Masuda, S., Kurosaka, T. \& Gotoda, H. Early detection of thermoacoustic combustion oscillations using a methodology combining statistical complexity and machine learning. Chaos 29, 103123 (2019).

${ }^{54}$ Sengupta, U., Rasmussen, C. E. \& Juniper, M. P. Bayesian machine learning for the prognosis of combustion instabilities from noise. In ASME Turbo Expo 2020: Turbomachinery Technical Conference and Exposition (American Society of Mechanical Engineers Digital Collection, 2020). 
${ }^{55}$ Komarek, T. \& Polifke, W. Impact of swirl fluctuations on the flame response of a perfectly premixed swirl burner. J.Eng.Gas. Turb. Power 132 (2010).

${ }^{56}$ Unni, V. R., Mukhopadhyay, A. \& Sujith, R. I. Online detection of impending instability in a combustion system using tools from symbolic time series analysis. Int. J. Spray. Comb. Dyn. 7, 243-255 (2015).

${ }^{57}$ Silva, C. F., Merk, M., Komarek, T. \& Polifke, W. The contribution of intrinsic thermoacoustic feedback to combustion noise and resonances of a confined turbulent premixed flame. Comb. Flame 182, 269-278 (2017).

${ }^{58}$ Murugesan, M., Singaravelu, B., Kushwaha, A. K. \& Mariappan, S. Onset of flame-intrinsic thermoacoustic instabilities in partially premixed turbulent combustors. Int. J. Spray. Comb. Dyn. 10, 171-184 (2018).

${ }^{59}$ Tony, J. et al. Experimental investigation on preconditioned rate induced tipping in a thermoacoustic system. Sci. Rep. 7, 1-7 (2017).

${ }^{60}$ Bonciolini, G. \& Noiray, N. Bifurcation dodge: avoidance of a thermoacoustic instability under transient operation. Nonlinear Dynamics 96, 703-716 (2019).

${ }^{61}$ Manikandan, S. \& Sujith, R. I. Rate dependent transition to thermoacoustic instability via intermittency in a turbulent afterburner. Exp. Therm. Flu. Sci. 114, 110046 (2020).

62 Pavithran, I. \& Sujith, R. I. Effect of rate of change of parameter on early warning signals for critical transitions. Chaos 31, 013116 (2021).

${ }^{63}$ Premchand, C. P., George, N. B., Raghunathan, M., Unni, V. R. \& Sujith, R. I. Lagrangian analysis of intermittent sound sources in the flow-field of a bluff-body stabilized combustor. Phys. Fluids 31, 025115 (2019).

${ }^{64}$ Yu, K. H., Trouvé, A. \& Daily, J. W. Low-frequency pressure oscillations in a model ramjet combustor. J. Fluid. Mech. 232, 47-72 (1991).

${ }^{65}$ Hong, S., Speth, R. L., Shanbhogue, S. J. \& Ghoniem, A. F. Examining flow-flame interaction and the characteristic stretch rate in vortex-driven combustion dynamics using piv and numerical simulation. Comb.Flame 160, 1381 - 1397 (2013).

${ }^{66}$ Lee Rodgers, J. \& Nicewander, W. A. Thirteen ways to look at the correlation coefficient. The Amer. Stat. 42, 59-66 (1988). 
${ }^{67}$ Moran, P. A. Notes on continuous stochastic phenomena. Biometrika 37, 17-23 (1950).

${ }^{68}$ Unni, V. R. et al. On the emergence of critical regions at the onset of thermoacoustic instability in a turbulent combustor. Chaos 28, 063125 (2018).

${ }^{69}$ Frenkel, D. Seeds of phase change. Nature 443, 641-641 (2006).

${ }^{70}$ Madjarova, V. D., Kadono, H. \& Toyooka, S. Dynamic electronic speckle pattern interferometry (despi) phase analyses with temporal hilbert transform. Optics express 11, 617-623 (2003).

${ }^{71}$ Pawar, S. A., Seshadri, A., Unni, V. R. \& Sujith, R. I. Thermoacoustic instability as mutual synchronization between the acoustic field of the confinement and turbulent reactive flow. J. Fluid. Mech. 827, 664-693 (2017).

\section{Data availability}

All the data presented in this paper are available from the corresponding author on reasonable request.

\section{Acknowledgements}

This research was supported by the Swarnajayanti Fellowship (Grant no:DST/SF/1(E C)/2006) and also by the J C Bose Fellowship (Grant no: JCB/2018/000034/SSC) awarded by the Department of Science and Technology (DST), Government of India. N.B.G acknowledges the financial support of the East Africa Peru India Climate Capacities project (18_II_149_Global_A_Risikovorhersage) funded by the Federal Ministry for the Environment, Nature Conservation and Nuclear Safety and the International Climate Initiative. E.S acknowledges the financial support by RFBR, project number 20-07-01071. The authors would like to thank Mr. Sundar Bharathi, Ph.D student from Chemical Engineering at IIT Madras, India for the helpful discussions in optimizing the code for parallel processing. The authors would like to thank Mr. Midhun. P.R., Ms. Reeja. K. V. and Mr. Thilagaraj. S. of Aerospace department, IIT Madras for their assistance in carrying out the experiments.

\section{Author contributions}

E.S. and R.I.S. conceived the project. M.R. and N.B.G. conducted the experiments with inputs from R.I.S and V.R.U. M.R., N.B.G. and V.R.U performed the data analysis guided by E.S., R.I.S and J.K. R.I.S and J.K supervised the study. All the authors participated in writing the manuscript. 


\section{Competing interests}

The authors declare no competing interests. 


\section{Supplementary Files}

This is a list of supplementary files associated with this preprint. Click to download.

- SupplementaryInformation.docx 